\title{
Poisson's ratio of two-phase composites
}

\author{
C.L. Hsieh, W.H. Tuan* \\ Department of Materials Science and Engineering, National Taiwan University, Taipei 106, Taiwan
}

Received 8 October 2004; received in revised form 6 January 2005; accepted 24 January 2005

\begin{abstract}
In the present study, a model to predict the upper and lower bounds for the Poisson's ratio of two-phase composites is proposed. Only the elastic modulus and Poisson's ratio of each component are needed in order to carry out the prediction. Experimental data from other studies were collected and compared with the prediction; reasonable agreement has been found.

(C) 2005 Elsevier B.V. All rights reserved.
\end{abstract}

Keywords: Composites; Modeling; Mechanical properties-elastic behaviour

\section{Introduction}

To predict the elastic constants of composites by using only the elastic constants of each component has attracted much attention [1-19]. However, most the predictions offer upper and lower bounds for the elastic constants $[4-6,8-15,18]$. It is mainly due to that the elastic constants depend on the shape of each phase $[6,8,10,16]$, phase continuity [17] and interface integrity [12-16] of composites. The presence of defects, such as pores, cracks, can also affect the elastic constants of the composites.

Poisson's ratio describes the extent of transverse strain as a body is under an external force. Among the available theoretical models on the elastic constants [1-19], the Poisson's ratio has attracted much less attention $[15,18]$. One may argue that the Poisson's ratio $(v)$ can be calculated by knowing the bulk modulus $(K)$ and shear modulus $(G)$ as

$v=\frac{3 K-2 G}{6 K+2 G}$

However, care has to be taken by using the above equation to calculate the upper and lower bounds for Poisson's ratio. As pointed out by Zimmerman [18], if the upper bound for both bulk modulus and shear modulus are used in Eq. (1) to calculate the upper bound for Poisson's ratio, the result is

\footnotetext{
* Corresponding author. Tel.: +886 23659800; fax: +886223634562.

E-mail address: tuan@ccms.ntu.edu.tw (W.H. Tuan).
}

misleading, as demonstrated in Fig. 1. The following equations should be used instead to obtain the correct upper bound (denoted with a superscript $u$ ) and lower bound (denoted with a superscript 1) for the Poisson's ratio [18].

$\nu_{\mathrm{c}}^{\mathrm{u}}=\frac{3 K_{\mathrm{c}}^{\mathrm{u}}-2 G_{\mathrm{c}}^{1}}{6 K_{\mathrm{c}}^{\mathrm{u}}+2 G_{\mathrm{c}}^{1}}$

$v_{\mathrm{c}}^{1}=\frac{3 K_{\mathrm{c}}^{1}-2 G_{\mathrm{c}}^{\mathrm{u}}}{6 K_{\mathrm{c}}^{1}+2 G_{\mathrm{c}}^{\mathrm{u}}}$

From Fig. 1, though the Poisson's ratio can be calculated by using the above equations, the upper and lower bounds are widely apart. It demonstrates that further refinement on the estimation of Poisson's ratio is needed. In the present study, a model is proposed to estimate the upper and lower bounds for the Poisson's ratio of two-phase materials.

\section{Modeling}

Previous models on elastic modulus often assumed implicitly that the Poisson's ratio of two phases are either 0 (no transverse constraint) or 0.5 (both phase incompressible) $[1,2]$. Owen and Koller had proposed a simple model to takes the transverse constraint into account [19]. Such constraint is essential to the estimation of Poisson's ratio. However, the model was used only to estimate the elastic modulus of twophase composite. In the present study, the same methodology 


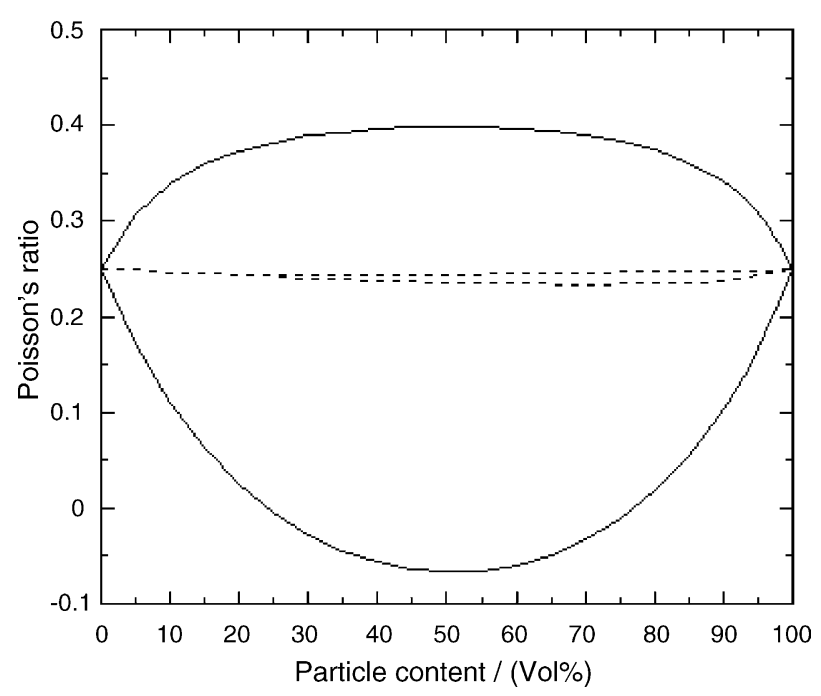

Fig. 1. The upper and lower bounds of Poisson's ratio as function of volume fraction. It assumes that the $K_{\mathrm{p}} / K_{\mathrm{m}}=G_{\mathrm{m}} / G_{\mathrm{p}}=20, v_{\mathrm{p}}=v_{\mathrm{m}}=0.25$. The solid lines are plotted by using Eqs. (2) and (3). The dotted lines are obtained by using upper bounds for both bulk and shear moduli in Eq. (1) or by using lower bounds for both moduli. Note that the two dotted lines cross each other.

is adopted to estimate the Poisson's ratio, the often-neglected issue.

Assuming that a two-phase unit is located within the matrix of a composite (Fig. 2). The matrix is a homogeneous medium that has the average properties of the composite. As a tensile stress, $\sigma$, is applied on the composite in the $z$ direction, both the matrix (denoted with a subscript $\mathrm{m}$ ) and particle (denoted with a subscript $\mathrm{p}$ ) share the applied stress as

$\sigma=\bar{\sigma}_{z}^{\mathrm{m}} V_{\mathrm{m}}+\bar{\sigma}_{z}^{\mathrm{p}} V_{\mathrm{p}}$

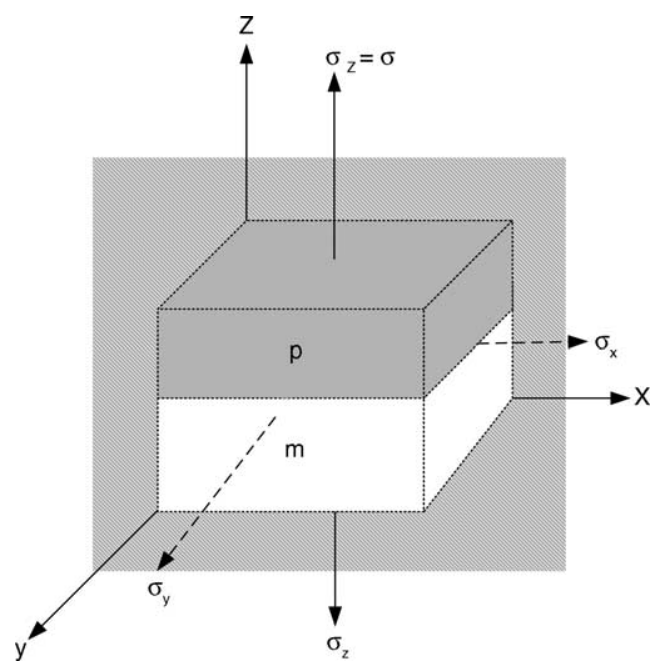

Fig. 2. One unit of two-phase material embedded in average homogeneous material and loaded by a tensile stress in $z$-direction. where $V$ is the volume fraction. The strain, $\varepsilon$, in the $z$-direction can be described as

$\varepsilon=\bar{\varepsilon}_{z}^{\mathrm{m}} V_{\mathrm{m}}+\bar{\varepsilon}_{z}^{\mathrm{p}} V_{\mathrm{p}}=\bar{\varepsilon}_{z}$

$\bar{\sigma}$ and $\bar{\varepsilon}$ are the average stress and average strain, respectively. Though there is no stress in the $x$ - and $y$-directions as,

$\sigma_{x}=\bar{\sigma}_{x}^{\mathrm{m}} V_{\mathrm{m}}+\bar{\sigma}_{x}^{\mathrm{p}} V_{\mathrm{p}}=0$

the transverse strain in the $x$ - and $y$-direction is still existed as,

$\varepsilon_{x}=\bar{\varepsilon}_{x}^{\mathrm{m}} V_{\mathrm{m}}+\bar{\varepsilon}_{x}^{\mathrm{p}} V_{\mathrm{p}}=\bar{\varepsilon}_{x}$

The transverse strain is the same in the $x$ - and $y$-directions.

For each two-phase unit, Fig. 2, the transverse strain is

$\bar{\varepsilon}_{x}^{\mathrm{m}}=\frac{1-v_{\mathrm{m}}}{E_{\mathrm{m}}} \bar{\sigma}_{x}^{\mathrm{m}}-\frac{v_{\mathrm{m}}}{E_{\mathrm{m}}} \bar{\sigma}_{z}^{\mathrm{m}}$

$\bar{\varepsilon}_{x}^{\mathrm{p}}=\frac{1-v_{\mathrm{p}}}{E_{\mathrm{p}}} \bar{\sigma}_{x}^{\mathrm{p}}-\frac{v_{\mathrm{p}}}{E_{\mathrm{p}}} \bar{\sigma}_{z}^{\mathrm{p}}$

where $E$ is the elastic modulus. The strains for each phase of the unit in $z$-direction are

$\bar{\varepsilon}_{z}^{\mathrm{m}}=\frac{-2 v_{\mathrm{m}}}{E_{\mathrm{m}}} \bar{\sigma}_{x}^{\mathrm{m}}+\frac{1}{E_{\mathrm{m}}} \bar{\sigma}_{z}^{\mathrm{m}}$

$\bar{\varepsilon}_{z}^{\mathrm{p}}=\frac{-2 v_{\mathrm{p}}}{E_{\mathrm{p}}} \bar{\sigma}_{x}^{\mathrm{p}}+\frac{1}{E_{\mathrm{p}}} \bar{\sigma}_{z}^{\mathrm{p}}$

The Poisson's ratio is the ratio of strain in the direction without load to that of the direction with load as

$v=-\frac{\bar{\varepsilon}_{x}}{\bar{\varepsilon}_{z}}=-\frac{\bar{\varepsilon}_{y}}{\bar{\varepsilon}_{z}}$

By using Eqs. (5) and (7), the above equation can be modified as

$v_{\mathrm{c}}=-\frac{\bar{\varepsilon}_{x}^{\mathrm{m}} V_{\mathrm{m}}+\bar{\varepsilon}_{x}^{\mathrm{p}} V_{\mathrm{p}}}{\bar{\varepsilon}_{z}^{\mathrm{m}} V_{\mathrm{m}}+\bar{\varepsilon}_{z}^{\mathrm{p}} V_{\mathrm{p}}}$

Then insert Eqs. (8)-(11) into the above equation, the following equation is obtained,

$$
\begin{gathered}
\left(\left(1-v_{\mathrm{m}} / E_{\mathrm{m}}\right) \bar{\sigma}_{x}^{\mathrm{m}}-\left(v_{\mathrm{m}} / E_{\mathrm{m}}\right) \bar{\sigma}_{z}^{\mathrm{m}}\right) V_{\mathrm{m}} \\
+\left(\left(1-v_{\mathrm{p}} / E_{\mathrm{p}}\right) \bar{\sigma}_{x}^{\mathrm{p}}-\left(v_{\mathrm{p}} / E_{\mathrm{p}}\right) \bar{\sigma}_{z}^{\mathrm{p}}\right) V_{\mathrm{p}} \\
v_{\mathrm{c}}=-\frac{+\left(\left(-2 v_{\mathrm{m}} / E_{\mathrm{m}}\right) \bar{\sigma}_{x}^{\mathrm{m}}+\left(1 / E_{\mathrm{m}}\right) \bar{\sigma}_{z}^{\mathrm{m}}\right) V_{\mathrm{m}}}{+\left(\left(-2 v_{\mathrm{p}} / E_{\mathrm{p}}\right) \bar{\sigma}_{x}^{\mathrm{p}}+\left(1 / E_{\mathrm{p}}\right) \bar{\sigma}_{z}^{\mathrm{p}}\right) V_{\mathrm{p}}}
\end{gathered}
$$

In the above equation, the subscript $\mathrm{c}$ denotes composite. The stress in the matrix and particle of the two-phase unit is the same as

$\bar{\sigma}_{z}^{\mathrm{m}}=\bar{\sigma}_{z}^{\mathrm{p}}, \quad \bar{\sigma}_{x}^{\mathrm{m}}=\bar{\sigma}_{x}^{\mathrm{p}}=0$

Therefore it is an iso-stress case. Eq. (14) can be simplified as

$v_{\mathrm{c}}=\frac{\nu_{\mathrm{m}} V_{\mathrm{m}} E_{\mathrm{p}}+v_{\mathrm{p}} V_{\mathrm{p}} E_{\mathrm{m}}}{V_{\mathrm{m}} E_{\mathrm{p}}+V_{\mathrm{p}} E_{\mathrm{m}}}$ 
Table 1

Elastic constants of the matrix and particle of several composites

\begin{tabular}{lrrrrrr}
\hline $\begin{array}{l}\text { Systems } \\
\text { (matrix-particle, m-p) }\end{array}$ & $E_{\mathrm{m}}$ & $E_{\mathrm{p}}$ & $E_{\mathrm{p}} / E_{\mathrm{m}}$ & $v_{\mathrm{m}}$ & $v_{\mathrm{p}}$ & Reference \\
\hline Co-WC & 207 & 700 & 3.4 & 0.31 & 0.19 & {$[20]$} \\
Epoxy-glass fiber & 3.5 & 73 & 21 & 0.35 & 0.22 & {$[21]$} \\
Polymer-glass & 1.7 & 76 & 41 & 0.44 & 0.21 & {$[22]$} \\
\hline
\end{tabular}

All the previous models assumed that the two phases are bonded together perfectly. By adopting the same assumption, the strain at the interface is therefore the same as

$\bar{\varepsilon}_{x}^{\mathrm{m}}=\bar{\varepsilon}_{x}^{\mathrm{p}}$

It thus is an iso-strain case. Since the stress in $x$-direction is zero, $\sigma_{x}=0$, Eq. (6) can thus change to

$\bar{\sigma}_{x}^{\mathrm{p}}=-\left(V_{\mathrm{m}} / V_{\mathrm{p}}\right) \bar{\sigma}_{x}^{\mathrm{m}}$

and the stress in $z$-direction is the same as the external stress.

From Eqs. (8) and (9), $\bar{\sigma}_{z}^{\mathrm{m}}$ and $\bar{\sigma}_{z}^{\mathrm{p}}$ can be expressed as the following equation

$\bar{\sigma}_{z}^{\mathrm{m}}=\bar{\sigma}_{z}^{\mathrm{p}}=\frac{\left(1-v_{\mathrm{m}}\right) E_{\mathrm{p}} V_{\mathrm{p}}+\left(1-v_{\mathrm{p}}\right) E_{\mathrm{m}} V_{\mathrm{m}}}{\left(v_{\mathrm{m}} E_{\mathrm{p}}-v_{\mathrm{p}} E_{\mathrm{m}}\right) V_{\mathrm{p}}} \bar{\sigma}_{x}^{\mathrm{m}}$

By inserting Eq. (19) into Eq. (14), then,

$$
\begin{aligned}
& {\left[\left(1-v_{\mathrm{p}}\right) E_{\mathrm{m}}-\left(1-v_{\mathrm{m}}\right) E_{\mathrm{p}}\right] V_{\mathrm{m}}+\left(v_{\mathrm{m}} V_{\mathrm{m}} E_{\mathrm{p}}\right.} \\
& v_{\mathrm{c}}=\frac{\left.+v_{\mathrm{p}} V_{\mathrm{p}} E_{\mathrm{m}}\right) X}{2\left(v_{\mathrm{p}} E_{\mathrm{m}}-v_{\mathrm{m}} E_{\mathrm{p}}\right) V_{\mathrm{m}}+\left(V_{\mathrm{m}} E_{\mathrm{p}}+V_{\mathrm{p}} E_{\mathrm{m}}\right) X}
\end{aligned}
$$

In the above equation, $X$ is

$X=\frac{\left(1-v_{\mathrm{m}}\right) E_{\mathrm{p}} V_{\mathrm{p}}+\left(1-v_{\mathrm{p}}\right) E_{\mathrm{m}} V_{\mathrm{m}}}{\left(v_{\mathrm{m}} E_{\mathrm{p}}-v_{\mathrm{p}} E_{\mathrm{m}}\right) V_{\mathrm{p}}}$

\section{Comparison}

Eq. (16) implies that the transverse strain in the matrix and in the particulate of a two-phase unit is independent of each other. Though it is only true that the Poisson's ratio of the particle is the same as that of the matrix, it also leads to the upper bound for the prediction. To be demonstrated later, the upper bound is indeed close to the phase with higher Poisson's ratio. Eq. (20) takes the stress-strain coupling at interface into account. The equation can thus be treated as the lower bound for the composites.

The experimental data are collected from various studies [20-22]. These systems were chosen for their elastic modulus ratio, $E_{\mathrm{p}} / E_{\mathrm{m}}$, covers a wide range, from 3.4 of the $\mathrm{Co}-\mathrm{WC}$ system to 41 of the polymer-glass system (Table 1). The upper and lower bounds as calculated by using Eqs. (16) and (20), respectively, are compared with the experimental data (Figs. 3-5).

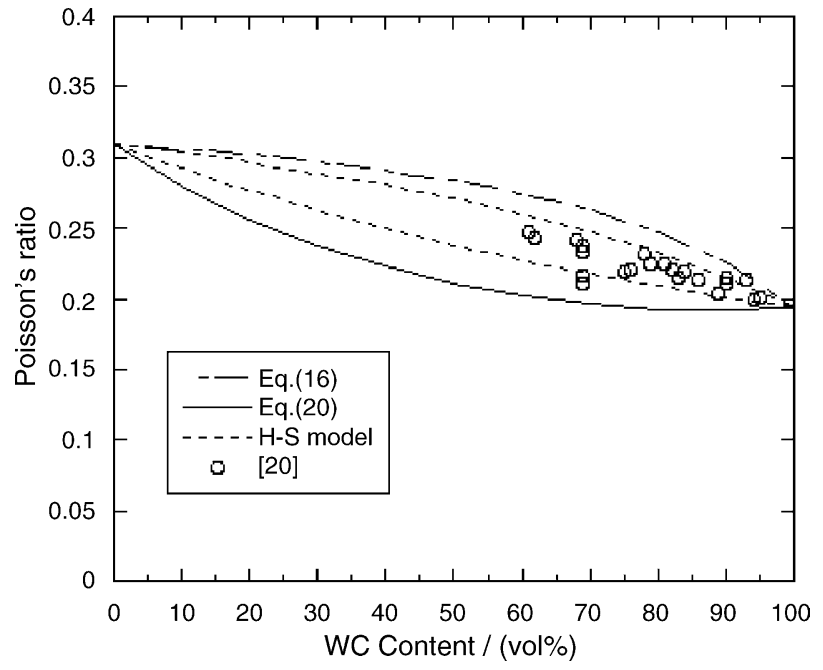

Fig. 3. Poisson's ratio of the Co-WC composites as function of WC content. The $E_{\mathrm{p}} / E_{\mathrm{m}}$ ratio of the system is 3.4 .

The upper and lower bounds proposed in the present study are also compared with other theoretical models. The most popular theoretical model to estimate the elastic modulus is the Hashin-Shtrikman (H-S) model [4]. However, the model only provided the bounds for bulk and shear moduli. The bounds on the Poisson's ratio can only be estimated by using Eqs. (2) and (3). The upper and lower bounds predicted by the modified $\mathrm{H}-\mathrm{S}$ bounds are also shown in the figures. Both the predictions proposed in the present study and that of the $\mathrm{H}-\mathrm{S}$ model cover most the experimental data. It demonstrates that the validity of both models. However, one should also note that the separation between the upper and lower bounds increases with the increase of $E_{\mathrm{p}} / E_{\mathrm{m}}$ ratio.

As the $E_{\mathrm{p}} / E_{\mathrm{m}}$ ratio is lower than 10 , the separation between the upper and lower bounds proposed in the present

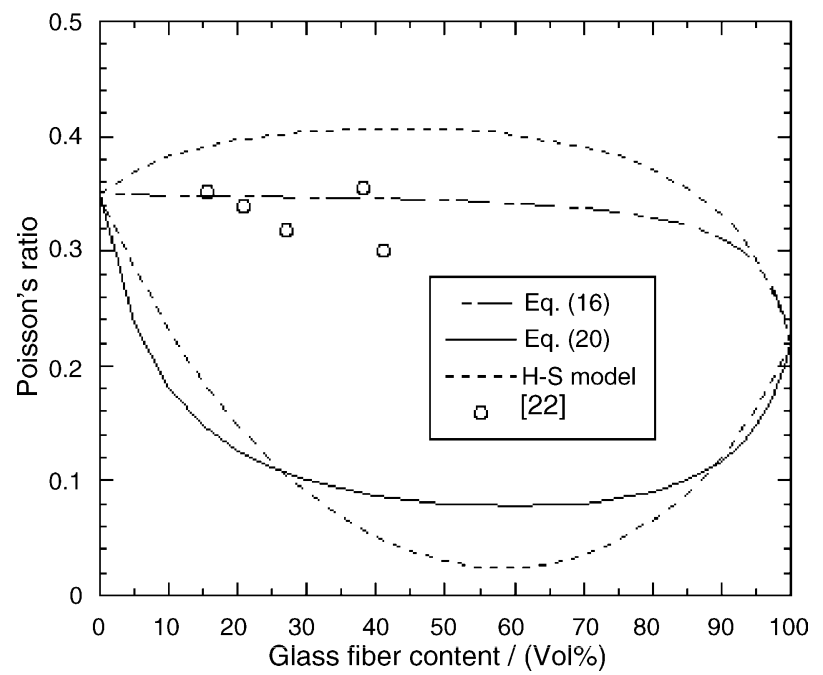

Fig. 4. Poisson's ratio of the epoxy-glass fiber composites as function of glass fiber content. The $E_{\mathrm{p}} / E_{\mathrm{m}}$ ratio of the system is 21 . 


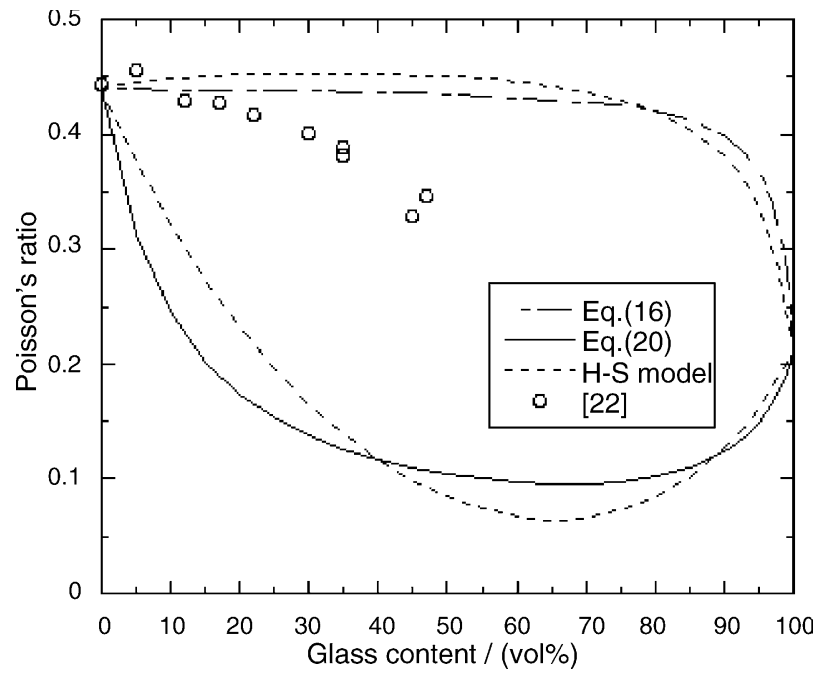

Fig. 5. Poisson's ratio of the polymer-glass composites as function of glass content. The $E_{\mathrm{p}} / E_{\mathrm{m}}$ ratio of the system is 41 .

study is slightly wider than those predicted by the $\mathrm{H}-\mathrm{S}$ model (Fig. 3). However, the upper and lower bounds as proposed in the present study can cover all the experimental data. As the $E_{\mathrm{p}} / E_{\mathrm{m}}$ ratio is higher than 20 , the separation between the upper and lower bounds proposed in the present study is slightly narrower than those predicted by the $\mathrm{H}-\mathrm{S}$ model (Figs. 4 and 5). Though the collection of experimental data is not comprehensive, it demonstrates that the proposed model can be applied to predict the Poisson's ratio of the two-phase composite with low $E_{\mathrm{p}} / E_{\mathrm{m}}$ ratio. As the $E_{\mathrm{p}} / E_{\mathrm{m}}$ ratio is large, the present model and $\mathrm{H}-\mathrm{S}$ model can only offer bounds with large separation.

The deviation between theoretical prediction and experimental data is till existed. Owen and Koller proposed that the stress concentration should be included into the model [19]. Our recent study demonstrates that the Poisson's ratio is more sensitive to the minor change in the microstructure characteristics [23]. It all demonstrates that further refinement on the model is still needed.

\section{Conclusions}

A model on the estimation of the Poisson's ratio of composite is proposed in the present study. The experimental data of three composite systems are compared with the model prediction. Agreement between the model prediction and the experimental data has been found. Comparison has also been made with the modified Hashin-Shtrikmam $(\mathrm{H}-\mathrm{S})$ bounds on Poisson's ratio. Similar to the $\mathrm{H}-\mathrm{S}$ bounds, the present model can offer close bounds on the composite with low $E_{\mathrm{p}} / E_{\mathrm{m}}$ ratio. However, as the $E_{\mathrm{p}} / E_{\mathrm{m}}$ ratio is large, the separation between the upper and lower bounds is also large.

\section{References}

[1] W. Voigt, Ann. Phys. 38 (1889) 573.

[2] A. Reuss, Z. Angew. Math. Mech. 9 (1929) 49.

[3] B. Paul, Trans. AIME 218 (1960) 36.

[4] Z. Hashin, S. Shtrinkman, J. Mech. Phys. Solids 11 (1963) 127.

[5] R. Hill, J. Mech. Phys. Solids 11 (1963) 357.

[6] B. Budiansky, J. Mech. Phys. Solids 13 (1965) 223.

[7] T.T. Wu, J. Appl. Mech. 32 (1965) 211.

[8] J.C. Halpin, J.L. Kardos, Polym. Eng. Sci. 16 (1976) 344.

[9] J.P. Watt, G.F. Davies, R.J. O'Connell, Rev. Geophys. Space Phys. 14 (1976) 541.

[10] Y. Benveniste, Mech. Mater. 6 (1987) 147.

[11] J.P. Matt, Phys. Chem. Miner. 15 (1988) 579.

[12] L.J. Walpole, J. Mech. Phys. Solids 14 (1966) 155.

[13] R.H.T. Yen, J. Appl. Phys. 42 (1971) 1101.

[14] G.W. Milton, N. Phan-Thien, Proc. Roy. Soc. London A 380 (1982) 305.

[15] K.S. Ravichandran, J. Am. Ceram. Soc. 77 (1994) 1178.

[16] T.T. Wu, Int. J. Solid Struct. 2 (1966) 1.

[17] C.L. Hsieh, W.H. Tuan, T.T. Wu, J. Eur. Ceram. Soc. 24 (2004) 3789.

[18] R.W. Zimmerman, Mech. Res. Commun. 19 (1992) 563.

[19] A.J. Owen, I. Koller, Polymer 37 (1996) 527.

[20] H. Doi, Y. Fujiwara, K. Miyake, Y. Oosawa, Metall. Trans. 1 (1970) 1417.

[21] O.I. Okoli, G.F. Smith, Comp. Struct. 48 (2000) 157.

[22] T.G. Richard, J. Comp. Mater. 9 (1975) 108.

[23] C.L. Hsieh, W.H. Tuan, Mater. Sci. Eng. A 393 (2005) 133. 